\title{
Effect of the interaction of MDP-based dentin bonding systems with chlorhexidine
}

The 10-MDP-based (10-methacryloyloxydecyl-dihydrogen phosphate) selfetching and universal systems are a remarkable category of bonding agents due to its chemical bonding capacity concealing interesting technical features with reduction of post-operative sensibility. Simultaneously to the advances on new monomers, biological knowledge evidenced the participation of dentin intrinsic proteolytic enzymes as matrix metalloproteinases (MMPs) and cysteine cathepsins (CCs), promoting the degradation of denuded collagen fibers not encapsulated by monomer resins in an adhesive process. On reverse of this process, strategies as the use of proteolytic inhibitors has been widely investigated and employed, in particular the chlorhexidine (CHX). However, the $\mathrm{CHX/MDP-based} \mathrm{systems} \mathrm{association} \mathrm{is} \mathrm{not} \mathrm{completely} \mathrm{elucidated} \mathrm{and} \mathrm{seems} \mathrm{to}$ impair the bonding-related properties. Thereby, investigating the effect of this interaction is important to prevent two important clinical strategies from cancelling out their benefits. In article 1 , the effect of $2 \%$ chlorhexidine digluconate on degree of conversion (DC) and bond strength (BS) were evaluated up to 6 months, using different dentin bonding systems (DBS): Adper ScotchBond MultiPurpose (MP) and Adper Single Bond 2 - SB (etch-and-rinse), Clearfill SE Bond-CSE and Clearfil Protect Bond-CPB (self-etching) and Adper Scotchbond Universal-SU (universal - self-etching mode). Regarding DC, CHX mostly affected the non-solvated DBS (MP and CSE), regardless of the main monomer. Concerning BS, CHX negatively affected MDP-based systems, especially universal system. For a better understanding about its action mechanism, in the article 2 , the use of 0.2 and $2 \%$ of chlorhexidine diacetate salt jointly with hydroxyapatite (HAP) have been proposed, once functional monomers chemically interact with hydroxyapatite from dentin, its molecular interaction also is affected, which may interferes with degree of conversion. DC and BS also were evaluated. The DBS used were MP, SU, CSE and CPB. DC and BS of MDPbased systems were impaired by $\mathrm{CHX}$ incorporation, mainly to CSE. HAP/CHX combination was able to restore $\mathrm{DC}$ values regarding control. Caution should be taken when both strategies (CHX and MDP) were used adjunctively for clinical bonding procedures. 